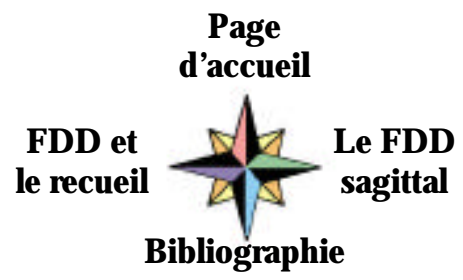

\title{
La notion de chaos et la géographie, quelques réflexions
}

Il y a au moins quatre bonnes sénies de raisons de s'interroger sur l'utilisation possible de la notion de chaos en géographie :

- Cette notion est appanue en physique et en mathématique, mais elle a été largement utilisée en chimie, mécanique, biologie non humaine. Des tentatives ont été faites en économie ou en histoire politique (cf. article de R. Passet dans Le Monde diplomatique de décembre 1989). La légitimité de ces transpositions, de cette nomadisation d'un concept, a fait l'objet d'un atelier lors du colloque du comité national du CNRS sur la transdisciplinarité.

- Des géographes se sont intéressés à la notion, ou à des notions voisines, et certains textes posent des problèmes de définitions. Y. Lacoste ouvre son demier livre par une réflexion sur le désordre et fait référence à l'œuvre de Coumot; 0 . Dollfus écrit sur les " chaos bornés » qui lui paraissent caracténiser la situation d'un certain nombre de pays ; il rejoint ainsi en partie J. Attali qui a écrit que « le désordre est l'état naturel du monde, la forme organisée y est l'exception ». A. Dauphiné a proposé une mise au point sur la notion (la publication de ce travail dans le présent numéro de L'E space géographique me dispense de développer les définitions de base à propos du chaos).

- On est frappé, à la lecture de la littérature sur la question, de la place qui est faite à des configurations spatiales ou des arrangements spatiaux, à côté des processus temporels. Comme la géographie s'efforce de décrire et de comprendre des arrangements spatiaux, on est tenté de voir dans quelle mesure la notion de chaos peut aider à en rendre compte, hors même du domaine de la météorologie (c'est-àdire en l'occurrence des configurations isobariques et de la disposition des flux aéniens).

- Les chaos ou situations chaotiques font leur apparition dans de nombreux articles qui utilisent des modèles dynamiques, dans les revues de langue anglaise, et avec des références explicites à la géographie.

Je voudrais traiter ici surtout des deux derniers points, c'est-à-dire de l'importance du chaos dans la typologie des configurations spatiales, et de leur place dans la modélisation. Il s'agit là de deux " entrées » différentes pour le même problème, de deux éclairages différents. Les deux points de vue se recouvrent certes largement, mais il m'a semblé utile de les distinguer dans un premier temps. 


\section{Le chaos parmi les configurations spatiales}

Les écrits sur le chaos font une lange place aux processus diachroniques, mais ils mentionnent aussi des configurations spatiales, et c'est par elles que je commencerai, pour deux raisons : d'une part, parce qu'elles nous concernent de façon privilégiée en tant que géographes; d'autre part, parce qu'il est bon de situer les chaos dans un ensemble d'états de l'espace ; un effort de clarification et de définition n'est pas inutile en la matière, car une certaine imprécision s'introduit dans le vocabulaire...

Toutes les situations ont en commun d'être caracténisées à différentes échelles, par ce qu'on ramènera par commodité à une opposition entre microétats et macroétats. Elles sont fondées sur l'interférence de systèmes de dimensions différentes, ou de systèmes avec les nombreux sous-systèmes qui les composent. Selon les caractères observés aux niveaux macro et micro, on peut distinguer un certain nombre d'états de l'espace :

- Le désordre est un état avec un grand nombre de degrés de liberté, donc un très grand nombre de dispositions micro, mais sans configurations identifiables à l'échelle macro. Par exemple, un corps visqueux ou liquide chauffé à la base peut connaître une intense agitation moléculaire qui correspond à la transmission de chaleur par conduction, sans que se développent des mouvements d'ensemble identifiables au niveau macro.

- Les « ordres» sont en général définis par l'existence de configurations macro, caractérisées par la répétition des structures, donc par une certaine autocorélation spatiale (on peut d'ailleurs en dire autant pour les processus temporels, avec l'existence de systèmes d'ondes). Pour reprendre le même exemple que ci-dessus, les cellules de Bénard ou les « rouleaux » qui apparaissent dans le corps visqueux quand l'échauffement à la base atteint une certaine valeur, opposent un ordre au désordre de la seule agitation moléculaire.

Il est courant de distinguer plusieurs types d'ordre, selon des critères descriptifs et/ ou génétiques :

- D'abord, on peut distinguer plusieurs niveaux d'ordre, selon les dimensions des macro-arrangements. D'un point de vue en quelque sorte génétique, ensuite, l'ordre peut représenter un état d'équilibre, ou une situation loin de l'équilibre, dans le cas des systèmes dissipatifs. On notera en passant que la notion d'équilibre est complexe, et qu'une certaine confusion règne dans le vocabulaire. Par exemple, il y a souvent des assimilations implicites entre la notion d'équilibre et d'équilibre stable, bien qu'il s'agisse de situations assez différentes. L'importance de l'équilibre pour l'explication des situations observables est telle que, un peu dans toutes les disciplines, les modèles d'équilibre ont souvent été privilégiés.

- Il existe des ordres fluctuants, coexistant avec des mouvements micro extrêmement actifs (comme dans le cas des cellules de Bénard), et des « ordres solidifiés » dont le cristal offre un exemple classique.

- Le chaos se différencie à la fois de l'ordre et du désondre. A la différence de ce demier, il présente des configurations identifiables, et un faible nombre de degrés de liberté. Mais il se différencie de l'ordre par l'absence d'autocorrélations spatiales et/ ou temporelles, par des fluctuations brutales et, dans les modèles, par le rôle des attracteurs étranges. Ceux-ci sont le résultat de l'action de processus antagonistes, dont certains tendent à faire converger les trajectoires, tandis que d'autres tendent à les faire diverger. Dans tous les processus, la sensibilité aux conditions initiales est 
forte. Dans les modèles de prévision, il y a une augmentation exponentielle des erreurs.

- Les processus chaotiques peuvent souvent être simulés par des équations très simples en apparence, et parfaitement déteministes, d'où la notion de " chaos déterministe ". D'autre part, les fluctuations chaotiques peuvent s'effectuer entre des valeurs limites, qui en fixent les bornes (chaos bornés).

On peut donc considérer que l'on est en présence de trois grands types "d'états", chacun, surtout les deux demiers, pouvant présenter un assez grand nombre de souscatégories.

Aussi, il est possible de porter un intérêt majeur à l'une ou l'autre de plusieurs lignes de clivage entre états différents. On ne peut considérer a priori une distinction comme essentielle, puisque l'on sera amené à privilégier l'une ou l'autre selon la nature du problème à résoudre. L'attention peut en particulier se porter sur :

- l'opposition entre les chaos et toutes situations non chaotiques, ordonnées ou désondonnées ;

- l'opposition entre les situations ordonnées, y compris les chaos, présentant des structures identifiables au niveau macro, et les désordres ;

- l'opposition entre les situations résultant de processus exponentiels, en quelque sorte " explosifs", et celles qui sont dues à l'action de processus conduisant à des évolutions qui tendent soit vers une valeur fixe, soit vers des oscillations entre des bomes, qu'elles soient régulières ou chaotiques. Assez curieusement, il semble que cette dernière opposition ait relativement peu retenu l'attention de certains auteurs de modèles. Pourtant, les situations " explosives " sont incapables de rendre compte de configurations ou d'évolutions durables, de par leur nature même. On pourrait même dire que les situations issues de processus exponentiels sont assez rarement observées, tout simplement parce qu'elles ne sont pas viables à long teme. C'est sans doute pour cette raison que l'on n'a pas beaucoup porté attention à cet aspect de la question. N égligence regrettable, car il n'est pas sans intérêt de s'interroger sur les conditions qui rendent possibles les situations observées, et qui les distinguent des situations non viables mais théoriquement envisageables.

Il semble bien que les évolutions réelles montrent des passages d'un état à un autre : du désordre à l'ordre (auto-organisation) ; d'un ordre à un autre (passage dont l'étude est une grande préoccupation des auteurs de modèles dynamiques) ; de l'ordre au chaos, ou du chaos à l'ordre. Les constructions d'ordre par fluctuation se situent donc dans des cadres assez différents.

Il semble que la grille de lecture dont la description vient d'être esquissée soit applicable à la géographie. Parce que celle-ci s'occupe de configurations complexes et des processus diachroniques qui leur donnent naissance ; parce qu'elle doit tenir compte des rapports entre microniveaux (par exemple, celui des décisions individuelles) et niveaux d'ordres de grandeur plus élevés ; parce que l'on rencontre souvent des situations qui ne sont pas désordonnées, sans présenter pour autant des structures simples, des autocorrélations nettes. Ce dernier point est très important, et il conduit à une réflexion sur les modèles et leurs rapports avec le chaos. 


\section{Les modèles et la complication du monde}

Le monde réel est à la fois varié et compliqué. Les modèles cherchent à en rendre compte par des simplifications, des schématisations, c'est leur essence même. Un écart est inévitable entre le monde réel-empirique et le monde des modèles; cet écart doit toujours être accepté, mais il y a bien des façons de le traiter, d'en rendre compte, ou de tenter de le réduire.

Une première solution consiste à considérer que le modèle rend compte de traits essentiels, et à traiter les résidus incompressibles - ceux qui demeurent quand la modélisation ne peut plus être amélionée - en temes différents de ceux du modèle, par exemple en termes qualitatifs si le modèle est quantitatif. Il n'est pas scandaleux de considérer que le modèle relève du monde des règles ou des lois, et que les résidus relèvent de celui de l'idiographique et du biographique, pour reprendre une distinction classique. La conception de la modélisation comme un guide vers une recherche monographique mieux informée est tout à fait légitime, et conduit à une démarche très pratiquée et très féconde. Il s'agit en d'autres temes de combiner le nomothétique et l'idiographique, souvent le quantitatif et le qualitatif.

Une deuxième solution consiste à introduire, à côté des composantes modélisées et déterminées, une "composante aléatoire ", qui prend en charge les variables négligées, les emeurs de mesure et les imprécisions des calculs. On connait bien la technique qui consiste à ajouter un "plus ou moins epsilon » comme demier terme des équations. On peut dans une certaine mesure considérer que cette présentation est une altemative paresseuse à la solution ci-dessus, mais elle n'est pas que cela. Elle est aussi une première façon d'introduire de l'aléatoire dans l'explication, introduction qui est, comme on dit, « incontournable » : un aléatoire « de premier genre ».

Dans les modèles non linéaires, la variation des conditions initiales et les fluctuations de paramètres autour de valeurs critiques conduisent à des bifurcations. Donc, le même modèle peut expliquer des situations très différentes, selon ces conditions initiales et les configurations de paramètres. Ces modèles à bifurcations permettent d'approcher la variété du monde réel, puisqu'ils peuvent simuler une grande variété de situations, et conduire de petites causes à de grands effets, ce qui les rend très réalistes. Ils sont plus efficaces pour expliquer la variété du monde que pour rendre compte de sa complication. Reste à savoir comment rendre compte des fluctuations des conditions initiales et des valeurs de paramètres. Elles peuvent être parfois rationalisées, mais on est souvent amené à les considérer comme relevant de l'aléatoire.

Mais on voit que l'aléatoire est ici introduit selon une autre perspective que celle qui a été mentionnée ci-dessus, et à une autre étape du raisonnement; c'est pourquoi je le qualifierai d'aléatoire "de deuxième genre ». Incidemment, l'aléatoire au niveau macro est souvent l'expression d'une fluctuation au niveau micro. Du point de vue qui nous intéresse ici, le fait que certains de ces modèles, comme ceux qui se rattachent à la " théorie des catastrophes", prennent en compte des bifurcations entre des équilibres, alors que beaucoup de modèles dynamiques sont concemés par des bifurcations entre des états "loin de l'équilibre", représente une distinction seconde, sinon secondaire. Dans tous les cas, il reste l'essentiel : des modèles déterministes comportant des éléments aléatoires rendent compte de la variété du monde, moins bien de sa complexité. 
Les modèles qui admettent des bifurcations vers des situations chaotiques présentent des analogies avec les modèles précédents, dans la mesure où ils offrent des possibilités de bifurcations en fonction de conditions initiales et de configurations de paramètres, avec la même amplification des petites fluctuations, relevant plus ou moins de l'aléatoire (du " deuxième genre »). Mais une différence, probablement très importante, vient du fait que, parmi les bifurcations possibles, certaines mènent à des situations chaotiques. Celles-ci présentent une grande complication, qui tendent souvent à suggérer un rôle important d'un aléatoire du type de celui qui a été défini comme l'aléatoire " de premier genre ». Or, il n'est est rien, puisque l'on sait que des équations simples et parfaitement déterministes peuvent, pourvu qu'elles soient non linéaires, conduire à des situations chaotiques.

Ainsi, les modèles qui incluent des bifurcations vers des situations chaotiques expliquent aussi bien que les autres la variété du monde, ils rendent mieux compte de sa complication, et ils étendent le champ du déteminé, donc, finalement, celui de la rationalité. Le schéma joint cherche à montrer leur place dans l'ensemble des modèles, en situant pour tous la part et la place de l'aléatoire par rapport à celle du déterminisme dans l'explication de la variété et de la complexité du monde.

\section{Sur la valeur heuristique}

Il est honnête d'enregistrer, pour finir, un certain nombre de limites à la valeur heuristique de la notion de chaos, de faire la part de quelques objections qui ont été faites. On peut les regrouper en trois thèmes majeurs :

- Les situations chaotiques n'apparaissent pas dans un assez grand nombre de modèles dynamiques. Rosser fait remarquer qu'il existe des séries de bifurcations qui « n'ont rien d'une cascade de Feigenbaum " (séries de bifurcations vers des situations chaotiques) et où « il n'y a nulle part de chaos nécessaire ».

- Pas mal d'essais de simulation, qui ont été faits avec des modèles " chaotiques", montrent que les configurations de paramètres qui aboutissent aux chaos sont assez rarement observées, plus rarement en tout cas que celles conduisant à des équilibres ou à des oscillations régulières, donc vers des attracteurs non étranges. May relève à ce propos un fait intéressant: dans ses études de terrain et de laboratoire sur des populations d'insectes, il a noté que les comportements chaotiques et oscillatoires caractérisent davantage les populations étudiées en laboratoire que celles qui ont été observées dans leur habitat naturel, et il explique cette observation par " the absence in laboratory conditions of many natural mortality factors ".

- Le chaos est une notion qui provient de la physique, et les hommes ne sont pas des molécules ou des atomes. 
On peut présenter à propos de ces objections quelques remarques rapides, mais il faudra y revenir. La demière objection me parait la moins pertinente. En effet, le chaos est une notion mathématique autant que physique; il est un caractère de certains types d'équations non linéaires (dira-t-on aussi « sans solution analytique » ?). Si ces équations sont pertinentes pour décrire des comportements humains ou sociétaux, il n'y a pas de raison de ne pas les utiliser, et de ne pas tirer des conclusions des simulations qu'elles produisent. $0 r$, cela semble bien être le cas. Des modèles qui combinent une fonction logistique et des fonctions des distances peuvent conduire à des comportements chaotiques. Or, ces fonctions sont tout à fait "réalistes ", puisqu'elles expriment simplement le fait que les croissances se font par la combinaison de boucles de rétroaction positives et négatives, et que la distance est un élément important pour la simulation des processus spatiaux. D'ailleurs, beaucoup de modèles dynamiques (ne faudrait-il pas dire même "la plupart des modèles») combinent ces deux types de fonctions (voir Allen, Wilson et quelques autres).

Figure 1: Déterminisme aléatoire et chaos dans les modèles

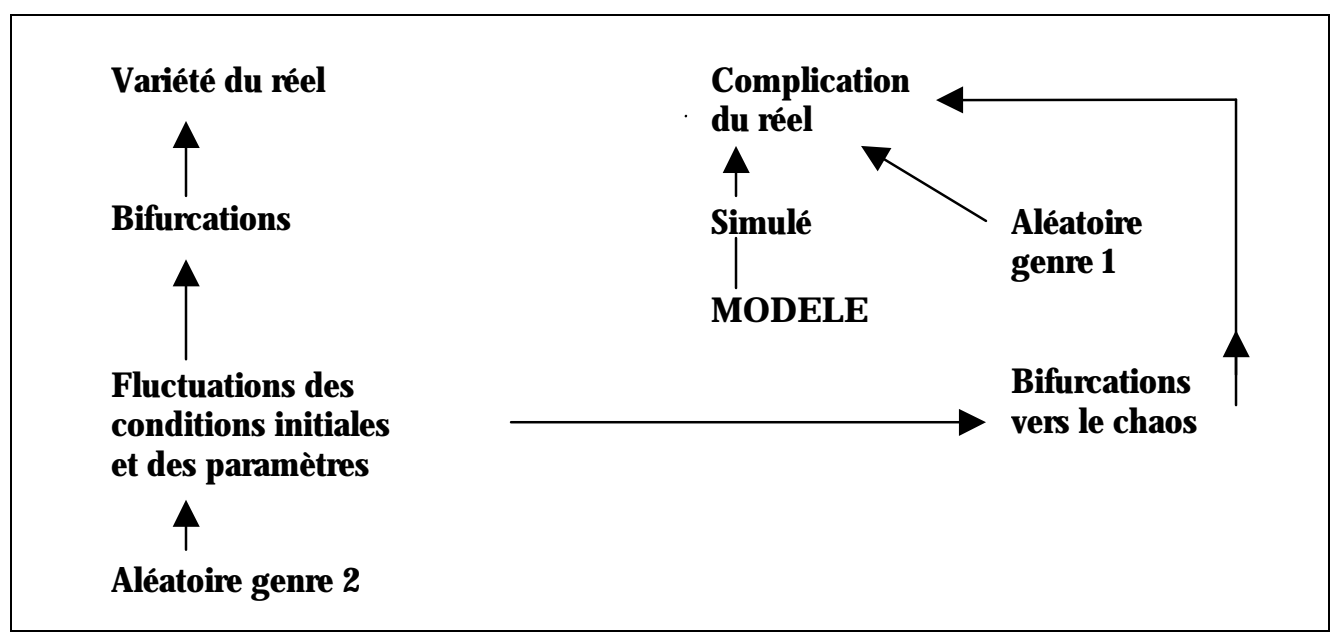

«L'irréalisme » des configurations de paramètres est bien plus important. En fait, il ouvre surtout une voie de recherche, parce qu'il demande à être testé sur de nombreux exemples, et aussi parce que le chaos peut toujours servir à poser des problèmes, à partir d'un cas limite théoriquement envisageable. Il y a là une analogie possible avec l'utilisation qui a été faite, en économie, de la notion d'équilibre. Celle-ci a été introduite par Pareto comme une notion heuristique puisque il s'est demandé si un certain nombre de mécanismes pouvaient mener à l'équilibre, en considérant comme seconde la question de savoir si cet équilibre est observé « dans la réalité » - on peut d'ailleurs dire qu'il serait étonnant que beaucoup d'équilibres évoqués en économie soient réalisés, puisqu'ils reposent sur une annulation des profits... Si l'on dispose d'un modèle qui met en ouvre des processus "réalistes", et que ce modèle conduit à des solutions chaotiques, il peut être fécond de se demander pourquoi, à la suite de quel filtrage, les situations chaotiques ne sont pas observées.

Bref, il me semble que tout ce qui précède montre qu'il y a quelque intérêt à s'occuper du chaos. On verra bien ce qu'il donnera. 